\title{
Le monde (de plus en plus) mouvant du cortex cérébral
}

La plasticité des connexions du système nerveux central est aujourd'hui un mécanisme admis du fonctionnement neuronal (voir $\mathrm{m} / \mathrm{s}$ $n^{\circ}$ 9, vol. 6, p. 840). L'étude de cette plasticité et de ses liens avec le comportement chez l'homme comme chez l'animal est devenue un domaine en plein développement dont les résultats s'accumulent. Parmi les travaux récents, trois retiennent l'attention parce qu'ils convergent vers une même conclusion, le rôle de la neuroplasticité dans l'élaboration, au long cours, d'un support neuronal de l'apprentissage.

Le premier travail vient d'être publié par Pascualleone et Torres (NIH, Bethesda, MD, USA) chez des aveugles entraînés à lire en Braille [1]. Les auteurs ont étudié la représentation corticale du doigt utilisé par ces personnes pour lire le Braille, c'està-dire l'étendue du cortex somesthésique dans lequel les neurones sont activés à partir du territoire cutané correspondant (voir $\mathrm{m} / \mathrm{s}$ Lexique Neurobiologie, mai 1992, p. 83). Les potentiels évoqués somesthésiques induits par la stimulation de l'index droit (doigt utilisé pour la lecture) étaient enregistrés sur un territoire sensiblement plus large que pour l'index gauche ("non lecteur") ou pour l'index de sujets contrôles. L'activation du cortex du côté opposé (grâce à la stimulation magnétique transcrânienne) bloque normalement les sensations induites par ces stimulations somesthésiques à partir d'une petite zone strictement localisée en miroir de la zone de réception. Chez les sujets aveugles, la zone à partir de laquelle cette inhibition était induite était, également, notablement élargie. Ces résultats plaident clairement en faveur d'une modification des connexions du cortex cérébral liées à lecture digitale. Ils concordent parfaitement avec ceux récemment publiés par l'équipe de Mike Merzenich (UCSF, San Francisco, CA, USA) lors d'analyses de la cartographie du cortex somesthésique chez le primate non humain [2-5]. Cette équipe travaille depuis des années sur les modifications des cartes de représentation corticale somesthésique lors de manipulations diverses des champs récepteurs (section d'un doigt, collage de deux doigts ensemble, etc.) (voir $\mathrm{m} / \mathrm{s}$ $n^{\circ} 6$, vol. 7, p. 624). Elle avait démontré, grâce à l'enregistrement électrophysiologique de nombreux neurones du cortex somesthésique, avant et plusieurs mois après manipulation des champs récepteurs somesthésiques, que l'étendue de la représentation corticale d'une région dépend de son utilisation. Le bien connu homunculus, dessiné par le neurochirurgien canadien Wilder Penfield dans les années 1930, subit des altérations internes - par extension ou restriction d'une représentation par rapport à d'autres - lorsque l'on modifie les domaines cutanés à partir desquels sont produits les messages nerveux. La dernière série de travaux de cette équipe franchit un nouveau pas dans cette démonstration, et même plusieurs. Elle démontre tout d'abord que les modifications de la représentation corticale sont inductibles à partir non pas seulement d'altérations des champs cutanés, mais aussi de l'utilisation normale des circuits nerveux. Des singes adultes ont été entraînés à détecter une différence de fréquence entre des stimulations tactiles répétées appliquées systématiquement sur le même doigt (figure 1). Au cours de cet apprentissage, les performances des singes se sont améliorées, la discrimination s'effectuant d'abord pour des stimulations distinctes de $6-8 \mathrm{~Hz}$ et baissant progressivement à $2-3 \mathrm{~Hz}$. Cet effet était sinon totalement restreint, du moins très largement prédominant au doigt entraîné, ce qui démontrait l'existence de modifications locales de l'organisation des réponses neuronales lors de l'apprentissage.

Ces modifications locales sont effectivement observées dans l'aire du cortex cérébral dans laquelle sont reçus les messages somesthésiques. Dans cette aire somesthésique, on peut établir une carte précise de la représentation des doigts en enregistrant de nombreux neurones. Les réponses obtenues permettent de dessiner les contours de petites régions dans lesquelles les neurones répondent à l'un ou à l'autre des doigts, ces régions étant elles-mêmes disposées suivant un ordre précis reproduisant l'organisation de la main (le $3^{\mathrm{e}}$ doigt entre le $2^{e}$ et le $\left.4 \mathrm{e} \ldots\right)$. Lorsque l'on enregistre les neurones chez un même singe à quelques mois d'intervalle, sans lui avoir fait subir d'apprentissage, on retrouve une organisation subrégionale extrêmement proche de celle définie originellement. Chez les singes entraînés, au contraire, on observe des modifications frappantes : la région contenant les neurones correspondant au doigt entraîné est nettement élargie alors que les régions voisines sont, au contraire, rétractées. Cette modification implique que des neurones qui jusque-là répondaient à un doigt ont cessé de le faire et ont, en revanche, été " recrutés" par le doigt voisin. Le mécanisme par lequel s'est effectué l'apprentissage (l'amélioration des performances comportementales du singe) implique donc le recrutement de populations neuronales jusque-là situées en dehors de la zone de réception des messages somesthésiques concernée par l'apprentissage.

$m / s$ n० 8-9 vol. 9, août-seplembre 93 


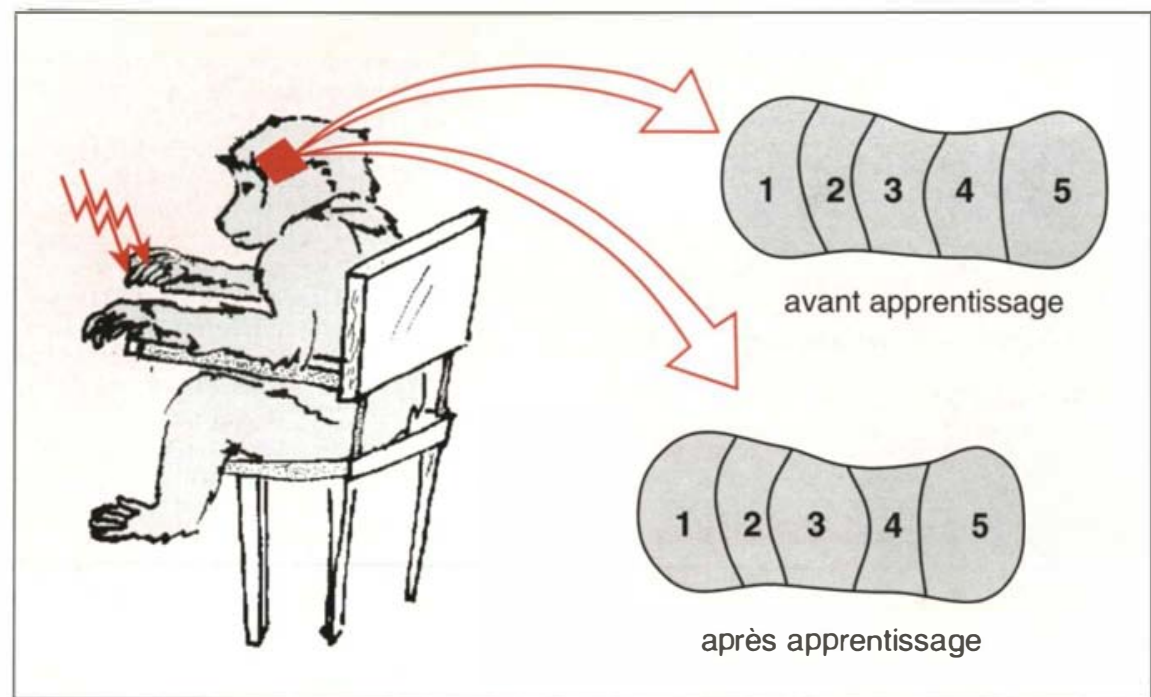

Figure 1. Principes de l'expérience de l'équipe de Mike Merzerich. Le singe maintenu en contention est soumis à une stimulation vibratoire d'un doigt lici le $3^{e}$ doigt de la main droitel. Pour obtenir une récompense, il doit discriminer la fréquence de la vibration. Au cours de l'apprentissage, il passe d'une discrimination de deux stimulations séparées par $6-8 \mathrm{~Hz}$ à celle d'une différence de seulement 2-3 Hz. Grâce à un volet crânien au-dessus de l'aire corticale somesthésique (rectangle), on enregistre les neurones répondant à des stimulations somesthésiques appliquées sur les doigts. La carte établie avant l'apprentissage (en haut) est profondément remaniée après celui-ci, le doigt entraîné gagnant du terrain sur ses voisins.

Cette extension se déroule également à d'autres niveaux du cortex cérébral. Chez le singe, comme chez l'homme, l'aire somesthésique est divisée en plusieurs zones plus particulièrement impliquées pour l'une (appelée $3 \mathrm{~b}$, celle dont il est question ci-dessus) dans la réception des messages induits par l'application d'une stimulation tactile cutanée, pour une autre (appelée $3 a)$ dans celle de messages induits à partir de récepteurs tendineux, etc. La stimulation répétée aboutissant à l'apprentissage était une stimulation cutanée, n'intéressant donc normalement pas 3a. L'enregistrement des neurones situés dans l'aire $3 \mathrm{a}$ a toutefois révélé l'émergence d'une zone aberrante de réponse cutanée. Le recrutement des neurones à l'intérieur même de $3 \mathrm{~b}$ s'accompagne donc d'une réorganisation complexe intéressant au moins une autre région du cortex cérébral.

La dernière partie de cette démonstration fort complète a abordé la question des mécanismes neuronaux de l'apprentissage: les neurones " entraînés " modifient-ils leur nombre de potentiels d'action ou un autre $\mathrm{m} / \mathrm{s} n^{\circ}$ 8-9 vol. 9, août-seplembre 93 tiale et temporelle des réponses neuronales. Les mécanismes subcellulaires impliqués dans cette réorganisation n'étaient pas dévoilés par ces expériences. Il était tentant, et c'est ce que faisaient les auteurs, d'y voir la conséquence d'une réorganisation des voies nerveuses corticales aboutissant à un renforcement des connexions synaptiques qui assurent le transfert des messages provenant de la région cutanée entraînée.

C'est effectivement un tel renforcement qui a été démontré par une analyse anatomique, particulièrement soignée, faite par le groupe d'Hiroshi Asanuma (Université Rockefeller, NY, USA). Dans ce travail, réalisé chez le chat adulte, des électrodes de stimulation ont été implantées dans le thalamus somatosensoriel, dernier relais des messages somesthésiques avant le cortex cérébral. Une stimulation répétée a été appliquée pendant quatre jours grâce à ces électrodes, réalisant une activation forcée de la voic somesthésique thalamo-corticale. Les animaux ont été ensuite sacrifiés et leur cortex cérébral, étudié en microscopie électronique (ce qui représente un travail considérable!). Le résultat a été à la hautcur de l'effort fourni puisque les autcurs amènent ainsi la première démonstration formelle d'un remaniement synaptique majeur induit par l'activité dans le système nerveux central adulte. Par rapport aux contrôles, il existait en effet une augmentation statistiquement significative du nombre des terminaisons synaptiques de la voie somesthésique $(+25,6 \%)$. De plus, la taille du contact synaptique formé par chacun des boutons était sensiblement plus grande ( + $28 \%)$. Tout cela s'accompagnait d'indices morphologiques d'une synaptogenèse active, comme la présence de nombreux polyribosomes à la base d'épines dendritiques (postsynaptiques) et l'existence de densités post-synaptiques perforées*. De façon intéressante, les connexions synaptiques étaient également modid'une liaison nette entre les deux phénomènes.

Au total, cette série d'expériences démontre que l'apprentissage, conduisant à une amélioration des performances de discrimination tactile, s'accompagne d'une modification spa-

- Densités post-synaptiques perforées : modification de
la structure de l'élément récepteur d'une synapse chi-
mique qui se traduit par une apparente interruption
de l'épaississement membranaire observé en microsco-
pie électronique. 
fiées dans l'aire motrice du cortex, suggérant un accroissement secondaire des voies intracorticales liant le cortex somesthésique et le cortex moteur.

La convergence des résultats obtenus dans ces trois séries d'expériences démontre ce que de nombreuses expériences suggéraient jusqu'à présent, à savoir le rôle fondamental joué par la neuroplasticité des connexions synaptiques dans le fonctionnement neuronal normal. La neuroplasticité sort ainsi brillamment du niveau cellulaire auquel la plupart des travaux la cantonnaient jusqu'à présent pour atteindre celui des systèmes intégrés et du comportement. La quatrième dimension du neurone devient celle du cerveau!

\section{Marc Peschanski}

Directeur de recherche à l'Inserm. CJF 91, 02, neuroplasticité et greffes intracérébrales, faculté de médecine, 8, rue du GénéralSarrail, 94010 Créteil, France.

\section{TIRÉS A PART}

M. Peschanski.

\section{RÉFÉRENCES}

1. Pascualleone A, Torres F. Plasticity of the sensorimotor cortex representation of the reading finger in Braille readers. Brain $1993 ; 116: 39-52$

2. Recanzone GH, Jenkins WM, Hradek GT, Merzenich MM. Progressive improvement in discriminative abilities in adult owl monkeys performing a tactile frequency discrimination task. I Neurophysiol $1992 ; 67: 1015-30$

3. Recanzone GH, Merzenich MM, Jenkins WM, Grajski KA, Dinse HR. Topographic reorganization of the hand representation of cortical area 3a. J Neurophysiol $1992 ; 67: 1031-56$.

4. Recanzone GH, Merzenich MM, Jenkins WM. Frequency discrimination training engaging a restricted skin surface in an emergence of a cutancous response zone in cortical area 3a. J Neurophysiol 1992; 67 : 1057-70.

5. Recanzone GH, Merzenich MM, Schreiner CE. Changes in the distributed temporal response properties of SI cortical neurons reflect improvements in performance on a temporally based tactile discrimination task. J Neurophysiol 1992; 67 : 1071-91.

6. Keller A, Arissian $\mathrm{K}$, Asanuma $\mathrm{H}$. Synaptic proliferation in the motor cortex of adult cats after long-term thalamic stimulation. J Neurophysiol $1992 ; 68$ : 295-308. 